\title{
Méthode actuelle de détermination du facteur Rhésus en contexte de transfusion et de grossesse
}

\author{
Willy Albert Flegel MD
}

Citation : CMAJ 2021 January 25;193:E124. doi : 10.1503/cmaj.201212-f

Voir la version anglaise de l'article ici : www.cmaj.ca/lookup/doi/10.1503/cmaj.201212

1

Il manque aux patientes de type Rhésus (Rh) négatif l'antigène $D$ à la surface de leurs globules rouges

Si elles reçoivent une transfusion de sang Rh positif, les patientes de type Rh négatif peuvent devenir immunisées contre l'immunoglobuline (Ig) anti- $D^{1}$. Ces patientes sont exposées à un risque de réactions transfusionnelles graves, et si elles deviennent enceintes, le fœtus pourrait présenter une anémie sévère. Un faible pourcentage de patientes $(0,5 \%)$ présente un phénotype sérologique $D$ « faible », et lorsqu'elles ont besoin d'une transfusion, les professionnels se demandent s'il faut leur administrer des culots globulaires de type Rh négatif et une prophylaxie par immunoglobulines anti-D (IgRh).

2

\section{Il existerait plus de 160 phénotypes moléculaires $D$ faible distincts}

Les types $D$ faible se regroupent selon l'origine ethnique. Les types 1 à 3 sont fréquents chez les personnes de race blanche, les variants de type 4 se retrouvent chez les personnes de race noire, et de type 15, le plus souvent chez les personnes asiatiques. Les autres types $D$ faible se rencontrent sporadiquement.

\section{3}

La détermination du type moléculaire $D$ faible améliore la sécurité des patientes sans augmenter les coûts

Même si la technologie qui permet de déterminer les types moléculaires existe depuis une vingtaine d'années, seul le test sérologique est appliqué de routine. L'établissement du type moléculaire est fiable, et cette approche de médecine de précision permet d'éviter des traitements superflus, mais doit faire l'objet d'une demande spécifique dans de nombreux hôpitaux².

4.

Les patientes qui présentent les 5 types $D$ faible les plus courants peuvent être traitées sans danger comme étant de Rh positif Les patientes porteuses des types moléculaires $D$ faible $1,2,3,4,0$ ou 4,1 devraient être traitées comme étant de Rh positif ${ }^{3}$. Les femmes enceintes porteuses de ces phénotypes $D$ faible ne tirent aucun avantage de la prophylaxie IgRh ${ }^{4}$. Elles ne devraient pas être exposées à cette immunoglobuline, qui provient de mélanges de plasma humain de milliers de donneurs immunisés. Cette approche permet de ménager les réserves limitées de sang Rh négatifs.

\section{Les patientes porteuses de types moléculaires $D$ faible moins courants devraient être traitées comme étant de Rh négatif}

Les types moins courants incluent le type $D$ faible 4,2; plus fréquent chez les personnes d'origine africaine ${ }^{6}$. Une liste exhaustive des types $D$ faible qui devraient être traités comme étant de Rh négatif est maintenue à jour par la Société internationale de transfusion sanguine ${ }^{7}$. Les professionnels qui hésitent face aux types à traiter comme étant de Rh positif ou négatif devraient communiquer avec leur laboratoire d'immunohématologie de référence.

\section{Références}

1. Flegel WA, Wagner FF, O'Donghaile DP. Anti-D immunization rates may exceed $50 \%$ in many clinically relevant settings, despite varying widely among patient cohorts. Transfusion 2020;60:1109-10.

2. Kacker S, Vassallo R, Keller MA, et al. Financial implications of RHD genotyping of pregnant women with a serologic weak D phenotype. Transfusion 2015;55:2095-103.

3. Flegel WA, Denomme GA, Queenan JT, et al. It's time to phase out "serologic weak D phenotype" and resolve D types with $R H D$ genotyping including weak D type 4 . Transfusion 2020;60:855-9.

4. Fung KFK, Eason E. No. 133-Prévention de l'alloimmunisation foeto-maternelle Rh. J Obstet Gynaecol Can 2018;40:e11-21.

5. Barty RL, Pai M, Liu Y, et al. RBCs: Where is universal donor blood being used? Vox Sang 2017;112:336-42.

6. Flegel WA, Denomme GA, Yazer MH. On the complexity of D antigen typing: a handy decision tree in the age of molecular blood group diagnostics. J Obstet Gynaecol Can 2007;29: 746-52.

7. Storry JR, Clausen FB, Castilho L, et al. International Society of Blood Transfusion Working Party on Red Cell Immunogenetics and Blood Group Terminology: report of the Dubai, Copenhagen and Toronto meetings. Vox Sang 2019;114: 95-102. 
Intérêts concurrents : Aucun déclaré.

Cet article a été révisé par des pairs.

Affiliation : NIH Clinical Center, National Institutes of Health, Bethesda, Md.

Financement : L'auteur bénéficie de l'appui du Programme intramural de recherche du NIH Clinical Center, ID ZIA CL002128.

Avis : Les opinions exprimées ici ne représentent pas nécessairement celles des National Institutes of Health, du ministère de la Santé et des Services sociaux ni du gouvernement fédéral américains.

Propriété intellectuelle du contenu : Il s'agit d'un article en libre accès distribué conformément aux modalités de la licence Creative Commons Attributions (CC BY-NC-ND 4.0), qui permet l'utilisation, la diffusion et la reproduction dans tout médium à la condition que la publication originale soit adéquatement citée, que l'utilisation se fasse à des fins non commerciales (c.-à-d. recherche ou formation) et qu'aucune modification ni adaptation n'y soit apportée. Pour plus de renseignements, consulter le https://creativecommons.org/licenses/ by-nc-nd/4.0/deed.fr.

Correspondance : Willy Flegel, waf@nih.gov

Le CMAJ vous invite à soumettre vos textes pour la rubrique "Cinq choses à savoir ... » en ligne à http://mc. manuscriptcentral. com/cmaj. 